\title{
Pericardiacophrenic Vein
}

National Cancer Institute

\section{Source}

National Cancer Institute. Pericardiacophrenic Vein. NCI Thesaurus. Code C53061.

The veins running parallel to the pericardiacophrenic arteries that drain into the brachiocephalic veins or superior vena cava. 